\title{
Automatic 3D reconstruction of indoor environment with mobile laser scanning point clouds
}

\author{
Yang Cui, Qingquan Li, Bisheng Yang, and Wen Xiao, Chi Chen, Zhen Dong
}

\begin{abstract}
D modelling of indoor environment plays an important role in various applications such as indoor navigation, BIM (Building Information Modelling), interactive visualization, emergency response, and so on. While automated reconstruction of 3D models from point clouds is receiving more and more attention. Indoor modelling remains a challenging task in terms of dealing with the complexity of indoor environment, the level of automation and restrictions of input data. To address these issues, an automatic indoor reconstruction method that quickly and effectively reconstructs indoor environment of multi-floors and multi-rooms using both point clouds and trajectories from mobile laser scanning (MLS) is proposed. The proposed automatic method of parametric structure modelling comprises of three steps. Firstly, structural elements, such as doors, windows, walls, floors, and ceilings, are extracted based on the geometric and semantic features of point clouds. Then, the point clouds are automatically segmented into disjoint segments likes rooms through a combination of visibility analysis and physical constraints of the structural elements, which ensures the integrity of the room-space partitions and yields priors for the definition of point cloud label for reconstructed model. Finally, 3D models of individual rooms are
\end{abstract}

Manuscript received (Write the date on which you submitted your paper for review.) This work was supported in part by the Key Program of the National Natural Science Foundation of China (No. 41531177), the National Science Fund for Distinguished Young Scholars of China (41725005), the National Key Research and Development Program of China (No. 2016YFB0502203), the National Natural Science Foundation of China (No. 41701530 and No. 41501427), China Special Postdoctoral Science Found (No. 2018T110802).

Y. Cui is with the Shenzhen Key Laboratory of Spatial Smart Sensing and Services \& The Key Laboratory for Geo-Environment Monitoring of Coastal Zone of the Nation al Administration of Surveying, Mapping and GeoInformation \& College of Information Engineering, Shenzhen University, Nanhai Road 3688, Shenzhen 518060, China (e-mail: cuiyang@ szu.edu.cn).

Q. Q. Li is with the Shenzhen Key Laboratory of Spatial Smart Sensing and Services \& The Key Laboratory for Geo-Environment Monitoring of Coastal Zone of the Nation al Administration of Surveying, Mapping and GeoInformation, Shenzhen University, Nanhai Road 3688, Shenzhen 518060, China (corresponding author phone: +86-755-2653-1934; e-mail: liqq@szu.edu.cn).

B. S. Yang is with the State Key Laboratory of Information Engineering in Surveying, Mapping and Remote Sensing, Wuhan University, Wuhan 430079, China (corresponding author phone: +86-27-6877-9699; e-mail: bshyang@whu.edu.cn).

W. Xiao is with the School of Engineering, Newcastle University, Newcastle Upon Tyne, NE1 7RU, UK (e-mail: wen.xiao@newcastle.ac.uk).

C. Chen and Z. Dong are with State Key Laboratory of Information Engineering in Surveying, Mapping and Remote Sensing, Wuhan University, Wuhan 430079, China (e-mail: chichen@whu.edu.cn; dongzhenwhu@whu.edu.cn) constructed by solving an energy optimization function via multi-label graph cuts. Three benchmark datasets from the International Society for Photogrammetry and Remote Sensing (ISPRS), collected by two handheld laser scanning (HLS) and a backpack laser scanning (BLS) system were used to evaluate the proposed method. Experiments demonstrate that the completeness and correctness of reconstructed surface models obtained by the proposed method are mostly larger than $60 \%$, and the average accuracy of model is close to $5 \mathrm{~cm}$.

Index Terms-3D reconstruction; Indoor modelling; Mobile laser scanning; Point clouds

\section{INTRODUCTION}

$I_{\mathrm{i}}^{\mathrm{N}}$ $\mathrm{N}$ recent years, three-dimensional (3D) reconstruction of indoor environment has drawn huge attention from the urban modelling community due to its applications in various fields, such as indoor navigation [1], architectural design [2], virtual reality [3], and real-time emergency response [4]. Traditional 3D indoor models are usually built up manually with commercial software, such as CAD, Revit and 3DReshaper. However, manually $3 \mathrm{D}$ reconstructed indoor modelling is a tedious, slow and expensive process [5]. To make this process more effective and efficient, many studies have focused on automatic generation of 3D indoor models over the past decade. However, robust 3D reconstructing of complex 3D indoor models still faces challenging issues [6] due to the restrictions in data, the complexity of spatial structures.

Recently, with the development of laser scanning technology and point cloud process technology, the automation level of 3D indoor modelling has been improved. Various types of laser scanning devices, such as consumer grade RGBD (RGB and depth) sensors, lightweight terrestrial laser scanners (TLS), and HLS or BLS, have been used for data acquisition of indoor scenes. RGBD images (e.g., Microsoft Kinect) acquired by a camera and a depth sensor are widely used in 3D visual applications, such as 3D virtual reality, 3D SLAM (simultaneous localization and mapping), robotic obstacle avoidance. However, RGBD sensors are limited by the small scanning range and high level of noise. TLS scanners can obtain high quality data, but suffer from low mapping efficiency because of the laborious scan station resetting and registration procedures. MLS systems can continuously obtain point clouds while moving around. They can be applied to indoor scenes that have complex layouts and can minimize the effects of occlusions. This is why easy-to-use and affordable indoor MLS 
systems are mostly used for data acquisition of large indoor scenes [7]. However, due to the complexity of indoor environment, point clouds data can be affected by many factors such as moving objects, multiple reflections and occlusions, which present dramatic challenges in model 3D reconstruction.

A number of approaches have been proposed for indoor modelling from point clouds. Some methods classify indoor point clouds and assign them with semantic labels, such as floors, walls, ceilings, and other objects [8], [9]. However, the resulting models have only semantic information but no structure attributes. More recent methods [6], [10], [11] focus on extracting plane and line elements to construct models. These methods treat the modelling as an issue of vector structure representation, without considering the semantic reconstruction of rooms. Therefore, other methods [12], [13] were proposed to construct 3D watertight models from extracted planes by graph-cuts to solve the issue. However, most of the methods are limited to TLS point clouds that are inefficient to acquire. Currently, more and more work [3], [14]-[19] have constructed indoor models using MLS systems, as they offer not only a less occluded point cloud but also a trajectory followed by the system during the acquisition process [18]. Nevertheless, the reconstruction methodologies based on MLS are highly dependent on data quality, integrity and are mostly applied to simple scenarios. Meanwhile, most of the approaches, e.g. [16], fail to consider the interconnected space recognition and modelling.

In order to address the shortcomings stated above, this study focuses on the combination of point clouds and trajectories from MLS to automatically 3D reconstruct indoor environment which can have multiple floors, multiple rooms and complicated connections. The method starts by structural elements (e.g., ceilings, floors, walls) detection based on the geometric features of point clouds. Then, the unorganized point clouds are split into meaningful rooms through a combination of visibility analysis and constraints of the structural elements, which only depends on geometric information of trajectory points and yields priors for the definition of point cloud label for reconstructed model. Finally, the 3D models of individual rooms are separately constructed by solving an energy optimization function based on multi-label graph cuts, which is formulated as a global optimization approach allows for the connectivity of walls and assignment of rooms to regions of the building.

The main contributions of the proposed method include, (1) an automatic semantic operator, which allows individual rooms to be modelled, is proposed to semantically label the point clouds of indoor environment with a combination of visibility analysis and physical constraints of the structural elements; (2) a multi-label graph cut is implemented to automatically $3 \mathrm{D}$ reconstruct indoor models of multi-floors and complicated connections from MLS point clouds. The reconstructed results include both volumetric solid models and surface models, which have detailed geometric, topological and semantic information of indoor space and is suitable for both 3D visualization and calculating purposes.

\section{RELATED WORK}

In the last decade, there are many approaches designed for indoor modelling by 3D laser scanning, and they mostly consist of two main steps: (1) point cloud semantic labelling, (2) 3D reconstruction of indoor environment

\section{A. Point Cloud Semantic Labelling}

Currently, there are two main strategies of point cloud semantic labelling. The first one is to assign each single point or voxel a label in the scene [20], [21]. Thomas [22] proposed a 3D semantic classification framework in which a set of geometric features are computed for every point from their multiscale spherical neighborhoods. Wang [6] used a learning framework of the Associative Markov Networks (AMNs) to assign each 3D point a label from a class set (floor, walls, roof, other objects). Armeni [9] proposed a semantic parsing method that parsed the whole building into disjoint spaces and learnt candidate windows' geometric and appearance features, then considered the context among semantic elements. Díaz-Vilariño [8] et al classified the point cloud to obtain the geometry of floors, walls and ceilings by intersecting planes according to their adjacency relationships. The final elements were detected by an energy function using the off-the-shelf LP/MIP solver and Structured SVM [23]. However, the learning progress needs a huge amount of training data. Rottmann [24] proposed a supervised learning approach to label different locations using boosting. The process needs to train a classifier using features extracted from vision and laser range data. The second strategy is to classify the point cloud depending on prior knowledge of segments [25]-[27]. Exemplar common methods are RANSAC-based plane fitting [28] and Hough transform-based line fitting [29]. Sanchez [30] classified point clouds into four classes (floor, ceiling, wall and others) from their point normal orientations. Michailidis [31] presented a new method to extract wall openings (windows and doors) of indoor scenes from point clouds. The method directly extracted windows and doors from a single wall surface. Previtali [15] proposed a methodology to detect openings based on voxelisation to distinguish 'occluded' from 'empty' regions in wall surfaces. In this paper, the structural elements, such as doors, windows, walls, floors, and ceilings, are detected by considering the geometric and semantic information of point clouds.

\section{B. 3D Reconstruction of Indoor environment}

Current methods for reconstruction of indoor environment are mainly line-based [6], [32], [33], plane-based [34], [35] and volume-based [36].

\section{Line-based reconstruction.}

Linear reconstruction is based on line frameworks to build indoor models. The lines are detected as intersections of extracted planes, followed by an extrusion assuming walls as planar and vertical surfaces [15]. Lin [33] presented an efficient 3D line segment grouping method, and introduced the "number of false alarms (NFAs)" into 3D point cloud to filter false positive detections. The unconnected lines contain excessive noise and detail. Wang [6] proposed a line extraction method 
using the conditional Generative Adversarial Nets (cGAN) deep learning method to obtain a regular line framework model that had topological structure. Liu [32] proposed a global analysis of the entire input point cloud by deep neural network (DNN) to detect primitive structures and reconstructed vector graphic floorplans from RGBD videos with camera poses. However, these methods could not express semantic information of rooms. Plane-based reconstruction

Some methods detect and reconstruct planes to represent indoor scenes. Plane-based reconstruction of indoor environment is similar to outdoor reconstruction [10]. The most widely employed model-fitting methods for plane segmentation are RANSAC and Hough transform from point clouds. The algorithms [30] generate unconnected planes from 3D point clouds to construct a model without spatial topological relationship. However, the methods are not robust in occluded and noisy indoor environment. Boulch [34] presented a method for constructing a piecewise planar approximation of an observed surface provided as range images. The method was generic and can be applied to any scene in man-made environments. However, the goal was not to reconstruct the most accurate surface, but to produce a simple piecewise-planar approximation and to reconstruct a whole building. Monszpart [11] proposed a simple, scalable, powerful approach to extract Regular Arrangements of Planes (RAP) from an unstructured and noisy raw scan to rebuild man-made scenes. This method can reconstruct large scale scene, but it requires long computing time.

Volume-based reconstruction

Volumetric reconstruction of indoor environment is based on some pre-defined rules for splitting and merging volumetric primitives. Oesau [37] detected the horizontal structures and vertical wall structures for 3D space partitioning. The final model was extracted from 3D cell decomposition by binary cell partitioning into either empty or solid space using graph-cuts. However, the method only built inside and outside partitions but did not model individual rooms, which was also the case for $\mathrm{Li}$ and Nan [36], [38]. Extensions of this method were attempted by further studies [12], [13], [17]. Ochmann [12], [39] proposed a method in which every TLS station was considered as the initial label for a room, and the reconstruction task was solved by energy minimization. The method relied on the prior knowledge of scanning stations, which caused over segmentations for long corridors. Mura [13], [40] reconstructed individual rooms in 3D environments with arbitrary wall orientations and the results outperformed the 2.5D reconstruction results of other approaches [12], [14], [17], [36], [38]. However, the method was only applied to small-scale scenes. Ikehata [41] reconstructed an indoor model as a structure graph, in which the nodes expressed structural elements such as rooms, doors, and objects, and the edges represented the geometric relationships. The main limitations were that the method relied only on local geometric information, and the modelling efficiency was low. Xiao [42] proposed a new method, "Inverse CSG", for building modelling in a Constructive Solid Geometry (CSG) consisting of volumetric primitives to exploit structural regularities. The method focused on large scene reconstruction without semantic information and scene understanding of the indoor environment.

The above reviews reveal that the binary space partition method [36]-[38] can be applied to partition the space into inside and outside by graph-cuts, however cannot label different rooms. Other state-of-the-art methods [12], [13], [39]-[41] can only be applied to TLS point clouds, resulting in limited usage in wider applications using low cost MLS systems. Some methods [3], [14], [16]-[19] are proposed to build multi-room models, depending on iterative heuristics or subsequent merging steps, using MLS point clouds. Other methods [6], [11], [42] for large scene reconstruction can only express the building's geometry and structural information, but are in lack of space recognition and semantic information. In this paper, the multi-story, multi-room, and complicated indoor apace models with detailed geometric, topological and semantic information are reconstructed.

\section{Methodology}

The flowchart of the whole framework is illustrated in Fig. 1 , showing the three key steps in the proposed method: structural element extraction, individual room segmentation, and structural model reconstruction. The structural elements of building interiors such as walls, ceilings, floors, doors, windows, and cylinders are extracted based on geometric and semantic features of point clouds. For multi-room segmentation, visibility analysis is carried out, including 1) simulating the visibility of point clouds from trajectory points based on the line-of-sight; 2) limiting the visible range of point clouds based on the locations of detected doors; and 3) visibility clustering for points with similar scanning trajectory. Finally, 3D structural models of individual rooms are separately constructed via multi-label graph cuts. In the results, the output 3D models consist of all ceilings, floors, walls, rooms, doors, windows and cylinders in the indoor environment.
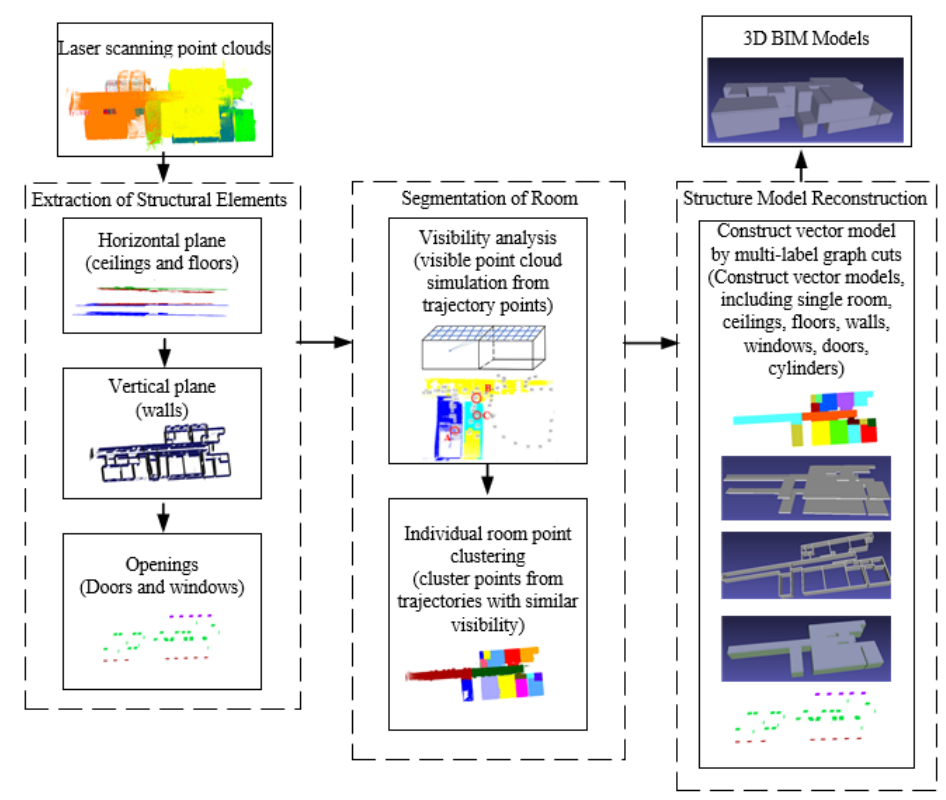

Fig. 1. Flowchart of 3D reconstruction of indoor environment from point clouds 


\section{A. Structural Elements Extraction}

\section{1) Horizontal and Vertical Plane Detection}

Real world indoor scenes are commonly Manhattan-world scenes, in which the major components of a building (walls, ceilings and floors) are composed of axis-aligned planes. The three dominant directions of a scene are generally identified by three strong peaks from the histogram of the normal distribution of point clouds [36]. Using the dominant directions, the point clouds can be transferred into a Manhattan coordinate frame. As the original data has no normal, they are approximated by the Principal Component Analysis (PCA) using K-nearest neighbors then used to determine the rotation parameters [43]. Finally, the point clouds are transformed into the Manhattan frame.

The transformation will facilitate planar surface detection from the point distribution along the axes. The density histogram of $\mathrm{z}$ coordinates is shown int Fig. 2(a) and the peaks in the histogram correspond to floors and ceilings. Peaks are extracted if the peak value is greater than a threshold $p_{v}$. If the distance between two peaks is less than a threshold $p_{d}$, the lower peak is eliminated. One example of extraction result is shown in Fig. 2(b). The blue planes represent the ceiling and floor of the first floor, and the red and green planes represent the ceiling and floor of the second floor. Note that the green plane is a part of the ceiling that is at a different height.

To extract walls from noisy point clouds, the RANSAC algorithm [28] is used to generate initial vertical planar surfaces. Points on horizontal planes will be discarded after the previous step and the rest points are mostly on vertical walls. There will be false detections in the initial RANSAC results which need refinement, as shown in Fig. 2 (c). To filter out false detections, a further step is proposed that iteratively refines these initial vertical segments. Specifically, the walls are restricted parallel to $x$ or $y$ axis as far as possible, by the following criteria: the $z$ component of the plane normal is less than a threshold $n_{z}$, and the slope of $x, y$ plane is less than a threshold $s_{x y}$. Planar segments are merged if the following conditions are satisfied: (1) the angle between the two planes is less than a threshold $\theta_{t}$,

(2) the distance from the centers of the two planes is less than a threshold $d_{t}$ [36]. These processes are repeated until all walls are refined, as shown in Fig. 2 (d). After that, parameters of every plane, including the normal $\left(n_{x}, n_{y}, n_{z}\right)$ and the distance $d$, are determined by PCA.

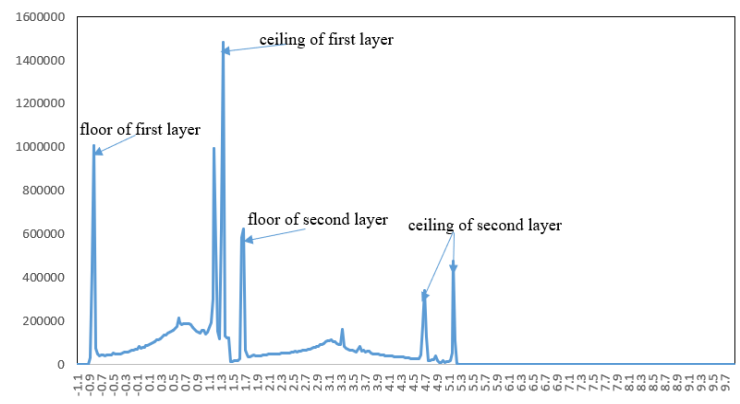

(a) z-coordinates histogram

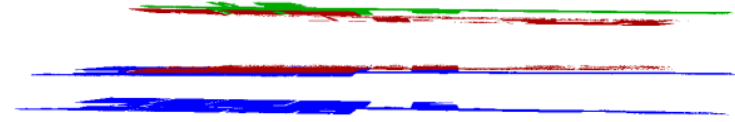

(b) Extraction of horizontal planes
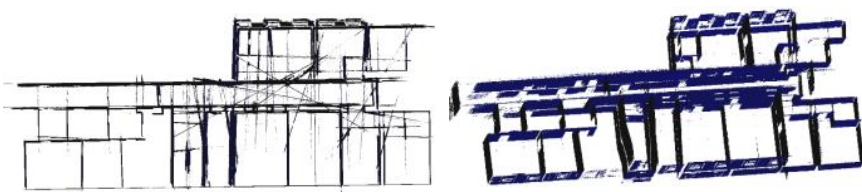

(c) The initial RANSAC segments

(d) The initial RANSAC segments

Fig. 2. Extraction of horizontal and vertical planes

\section{2) Opening Detection}

For indoor modelling, doors and windows can be considered as openings in segmented walls. It can be assumed that the bottoms of door openings are located on the floors, and similarly, a window has a certain height distance from the floor. Horizontal cross sections of the point clouds at certain heights from the floor are used to detect openings by identifying the number of points. In order to detect doors, point clouds are sliced horizontally into a series of sections at different heights, as shown in Fig. 3(a). The point clouds of sections at certain heights (e.g. $H_{\text {floor }}+0.1 m \sim H_{\text {floor }}+0.3 m$, $H_{\text {ceiling }}-0.3 m \sim H_{\text {ceiling }}-0.1 m, H_{\text {floor }}, H_{\text {ceiling }}$ are heights of the floor and ceiling) are projected onto a horizontal 2D grid, of which the cell size is $C_{s}$. Some sections will have no points at the opening of a door, but others will have points on top of the door. This difference can be used to detect the locations of doors, as shown in Fig. 3(b). The point clouds at the locations of doors are converted to a binary image, in which the pixel value is either 1 or 0 , meaning there are some or no points, respectively, as shown in Fig. 3(c). The initial line segments, which represent footprints of doors, are extracted by Hough transform from the binary image. Then nearby line segments are merged if the following conditions are satisfied: (1) the angle between the segments is less than a threshold $\theta_{a}$, (2) the distance from the centers of segments is less than a threshold $d_{c}$. Finally, door polygons are regularized by extruding line segments to a certain height and width, such as $h_{d}=2.2 \mathrm{~m}$ and $l_{d}=1 \mathrm{~m}$ according to the average door size of the indoor scene, The extraction of windows is similar to that of doors, and the only difference is the height value of selected horizontal cross-sections, the openings are shown in Fig. 3(d), in which the 
red ones are windows, and the green ones are doors, the purple ones are windows falsely detected as doors.

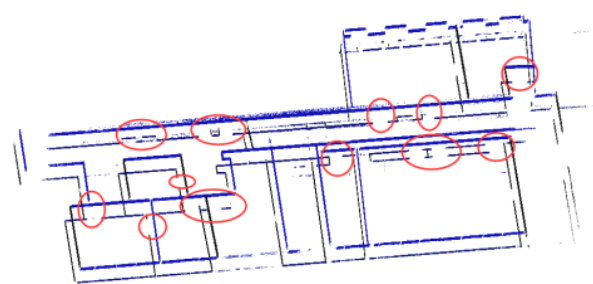

(a) Split point clouds from different heights (red circles are locations of doors)

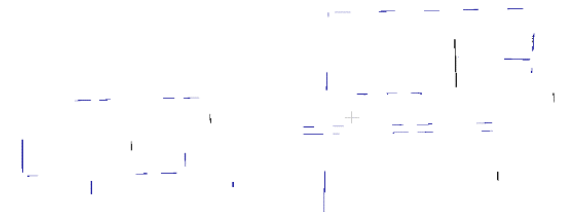

(b) Point clouds on top of door openings

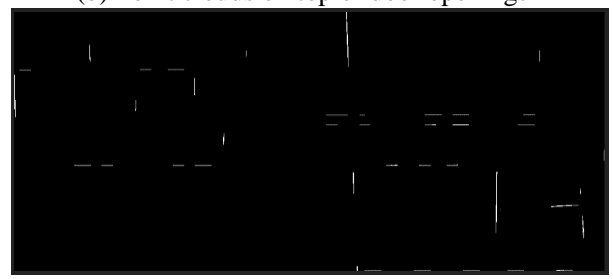

(c)The conversion of projected points into a binary image

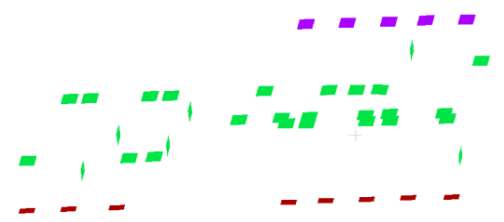

(d) The detected polygons in 3D representing doors Fig. 3. The detection of doors

\section{B. Individual Room Segmentation}

Ochmann [12] and Mura [13] used TLS points in a specific room based on the prior information of the scanner's position to segment rooms. Their methods are designed for TLS point clouds, but not for MLS point clouds. Inspired by their work, segmentation of rooms from unorganized point clouds are achieved by simulating visible point clouds from the trajectory of MLS based on the line-of-sight and clustering the points with similar scanning trajectories.

\section{1) Visibility Analysis}

Díaz-Vilariño [44] proposed a method that a voxel-based structure is designed for the creation of 3D Isovists. The point cloud is discretized in a voxel-based structure and voxels are identified as 'exterior', 'visible, 'occupied'. In this paper, the visibility of scanned points and previously detected door locations are used to subdivide the point cloud into individual rooms. During data acquisition, points are observed along the line-of-sight of the laser scanner, so they are considered as visible from corresponding trajectory point. In order to improve the computing efficiency, the segmented planes of indoor scenes are divided into uniform grids, so the visibility of all points in one grid cell is analysis together as a patch. There is no need to simulate the visible points from the whole trajectory, so one of every 200 trajectory points is sampled from the original trajectories, as shown in Figs. 4 (a) and (b). However, for the MLS dataset without trajectory points, it needs to simulate the possible position of the scanner. First, the elevation of the scanner is obtained by averaging the elevations of the floor and ceiling, so that the height of the scanner is approximated. Then, the horizontal positions of the scanner are simulated by sampling points uniformly at a regular distance of $2 \mathrm{~m}$, so that each room will have at least one sample position. Visibility is analyzed by ray tracing along the sampled trajectory points and cells' center points of each plane, as shown in Figs. 4 (c) and (d).

The intersections between rays and all other segmented planes are along the line-of-sight. Points in the cell are considered as invisible if the cell center point is obstructed. Let $s$ be the ray between a trajectory point $p_{1}$ and a plane cell center point $p_{2}$. If the ray is intersecting with a segmented plane, noted as $p_{3}$ in Fig. 4 (c), the number of points around $p_{3}$ will be checked. Let $p_{3}$ be the center of a sphere and let the radius be four times of the plane point density value (the point clouds captured by different scanners have different densities).

If there is no point in the sphere, points in the cell of $p_{2}$ are considered visible, otherwise invisible. If the ray is not intersecting with any segmented plane, points in the cell are certainly visible, as shown in Fig. 4 (d). Fig. 4(e) shows the simulated visible point clouds of three trajectory points. The visible region changes (showing in different colors) from different trajectory points (red circle).

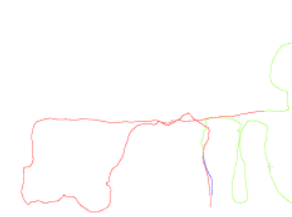

(a) Original trajectory points

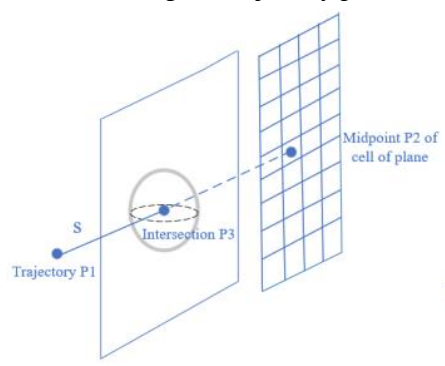

(c) $p_{3}$ is located in the segmented plane (d) The ray is not intersecting with any segmented plan

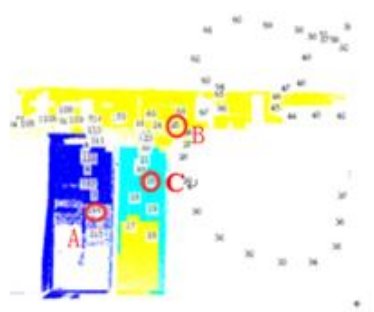

(e) The visible point clouds of three trajectory points A, B and C

Fig. 4. visibility analysis and visible point cloud diagram of trajectory points

As shown in Fig. 4 (e), some points in yellow can be observed from different rooms because of openings such as doors. Fig. 5 (a) shows an example when the scanner is near a 
door. A room can contain several doors in complicated indoor scenes, as shown in Fig. 5 (b). In fact, the doors can be used to separate rooms or corridors by limiting the range of visible points. An intuitive solution is depicted in Fig. 5 (c). For each sampled trajectory point $p_{k}$, doors that are visible to this point will be detected, then point clouds behind the doors will be considered invisible. To detect visible doors, a line segment between trajectory points $p_{i}, p_{i+1}$ on each side of a door $o_{j}$ will be intersected by the door, and the intersection is denoted as $I p_{j}$, where $I p_{j} \in I p, j=1, \ldots, m$ ( $m$ is number of doors). If the intersection $I p_{j}$ is visible from $p_{k}$, the door is considered visible from $p_{k}$. The visibility is then analysed similarly to the laser scanning point clouds. Each segmented wall plane $v w_{i} \in W, i=1, \ldots,|W|$ ( $W$ is all segmented wall group) is assessed if it will intersect with the ray between $I p_{j}$ and $p_{k}$. If so, $v p_{i}$ is the intersection, as shown in Fig. 5 (c) (left), in which $v p_{i}$ is treated as a sphere center and $R$ is a given radius. Then the number of points in the sphere is computed. If the number exceeds a threshold $N$, the intersection point $I p_{j}$ is invisible, meaning that the door is not visible from trajectory point $p_{k}$. Finally, the coverages of trajectory points are limited by the locations of detected doors, as shown in Fig. 5 (d).
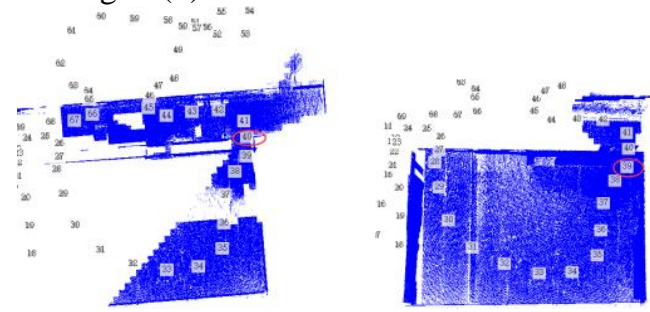

(a) Visible point clouds near a door opening

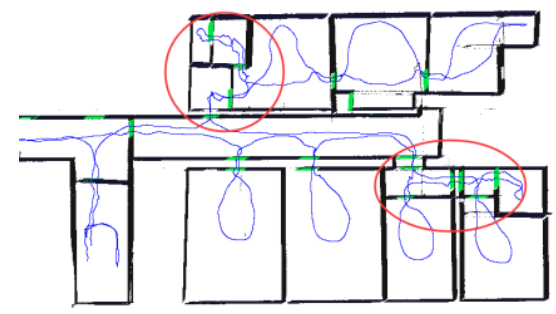

(b) The doors near sampled trajectory point
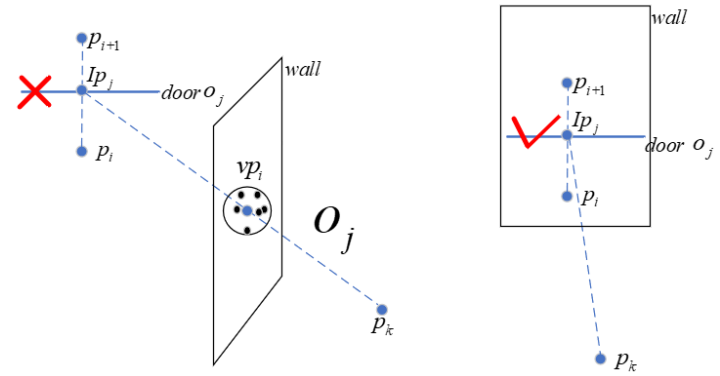

(c) Determining the doors near every trajectory point
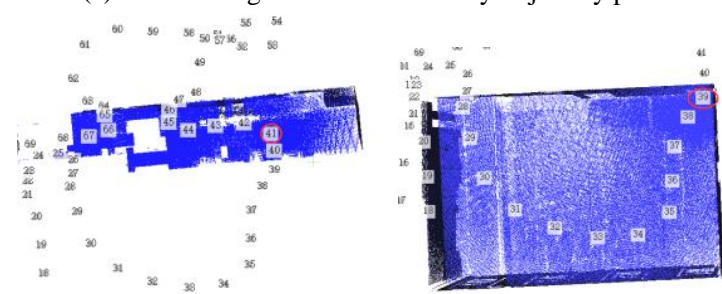

(d) updating the coverage of trajectory points

Fig. 5. The diagram of updating the coverage of trajectory points

2) Room Clustering

The visibility analysis is used to determine the scope of point clouds of every trajectory point. Trajectories in the same room will have overlapping point clouds, so individual room can be segmented by clustering overlapping points. In visibility analysis, the segmented planes of indoor scenes are divided into uniform grids. Therefore, the overlap between the visible grids of two trajectory points is calculated according to the index number of the same cells. Let $V_{T i}$ denote the grid cells of a sampled trajectory point $i$, and let $\operatorname{Overlap}\left(V_{T i}, V_{T j}\right)$ be the overlapping cells between $V_{T i}$ and $V_{T j}$, then the ratio of overlapping can be expressed as (1). For each trajectory point, the overlapping ratios will be computed with all the other trajectories to represent the similarity between trajectories.

$O V_{T i_{-} j}=\frac{\operatorname{Overlap}\left(V_{T i}, V_{T j}\right)}{V_{T i}}, j=1,2, \ldots i-1, i+1, \ldots, n$

The overlapping ratio $O V_{T_{-} j} \in[0,1]$ as defined. The more the value is, the more common scanning points the two trajectory points can observe. Therefore, an overlapping ratio threshold can be determined to obtain similar trajectory groups in which trajectory points have overlapping visibility. The OTSU algorithm [45] (Maximum Between-cluster Variance) is used to automatically adapt the overlapping ratio threshold to different datasets. The algorithm has a very wide range of applications in image segmentation for its simplicity and intuitiveness. Based on this method, the overlapping ratio is regarded as a value of a gray image, rescaled to [0,255], and the optimal threshold is sought for getting similar trajectory groups.

Algorithm 1 shows an overview of the clustering of point clouds from similar scanning trajectories for room segmentation. For each trajectory point $i$, the overlapping ratios with all the other trajectory points are computed as aforementioned. If overlapping ratio $O V_{T i_{-} j}, O V_{T j_{-} i}$ of two trajectory points $i, j(i, j=1,2 \cdots, n ; i \neq j)$ are both greater than the adaptive threshold value $t_{\text {adapted }}$, trajectory $j$ is considered similar to trajectory $i$ and is added to the trajectory group $i_{\text {Group }}$. Then the similar trajectory group $i_{\text {Group }}, i=1, \ldots, n$ of each trajectory point can be obtained. Next, if the number of common trajectory points in groups $i_{\text {Group }}$ and $j_{\text {Group }}$ is greater than a 
threshold $M_{\text {thread }}$, trajectory $i$ and $j$ will be merged, meaning the point clouds of these two will be clustered together. In the end, a set of clusters of point clouds that represent segmented rooms $R=\left\{R_{1}, \ldots, R_{\text {Nrooms }}\right\}$ are obtained. Fig. 6 (b) shows segmented rooms in different colors.

\begin{tabular}{l}
\hline Algorithm 1: Merging similar trajectories \\
\hline Input: overlapping ratios of any two trajectory points \\
$\left(O V_{T i_{-} j}, i, j=1,2 \cdots, n ; i \neq j\right) ;$
\end{tabular}

Output: merged similar trajectory groups for room segmentation

for $i=1$ to $n$ (total number of trajectory points)

$$
\begin{aligned}
& \text { for } j=1 \text { to } n(j \neq i) \\
& \text { if }\left(O V_{T i_{-} j}>t_{\text {adapted }} \& \& O V_{T j_{-} i}>t_{\text {adapted }}\right) \\
& \quad i_{\text {Group }}\{i\} \leftarrow j \\
& \text { end if } \\
& \text { end for }
\end{aligned}
$$

end for

for $i=1$ to $n \& \& i \notin R\{\} / / R$ is the final trajectory cluster.

$$
\begin{aligned}
& \text { for } j=i+1 \text { to } n \\
& j_{\text {Group }}=i_{\text {Group }} \wedge j_{\text {Group }} \\
& \text { if }\left(\text { num }\left(i j_{\text {Group }}\right)>\operatorname{num}\left(i_{\text {Group }}\right) * M_{\text {thread }} \& \&\right. \\
& \text { num } \left.\left(i j_{\text {Group }}\right)>\operatorname{num}\left(j_{\text {Group }}\right) * M_{\text {thread }}\right) \\
& \quad R\{i\} \leftarrow j / / \text { merged similar trajectory groups }
\end{aligned}
$$$$
\text { end if }
$$$$
\text { end for }
$$

end for

$$
\text { return } R=\left\{R_{1}, \ldots, R_{\text {Nrooms }}\right\}
$$

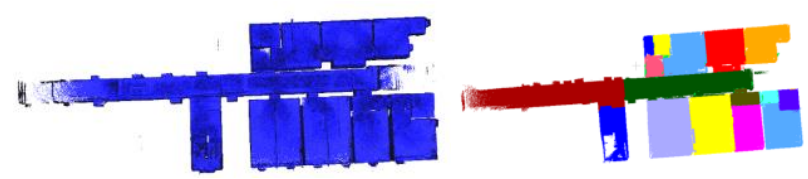

(a) Point clouds before room segmentation (b) Point clouds after room segmentation

Fig. 6. Point clouds before and after segmentation

\section{Structural Model Reconstruction}

The previous steps will segment the point clouds into individual room clusters $R=\left\{R_{1}, \ldots, R_{\text {Nrooms }}\right\}$, but the segmented results are only expressing room semantic information, lacking of geometric and structural attributes. In the last step, the final structural models are reconstructed using multi-label graph cuts, for which the segmented rooms and extracted walls are used as semantic constraints. Firstly, the segmented walls are projected to a 2D floorplan and fitted with linear primitive candidates, which will partition the ground plan into $2 \mathrm{D}$ cells $c_{i} \in\left\{c_{1}, \ldots, c_{n}\right\}$, as shown in Fig. 7 (a). The segmented rooms are used as semantic labels, as shown in Fig. 7 (b), and each cell is assigned a label from set $\left\{l_{1}, \ldots, l_{\text {Nrooms }}, l_{\text {out }}\right\}$, which includes one label for each room plus an additional label $l_{\text {out }}$ for the outer space. Meanwhile, the segmented walls are used to separate cells from adjacent rooms, of which cell labels should be different. The problem is expressed as an energy function, as shown in (2), that is minimized by multi-label graph cuts [18], [46]-[48].

$$
E_{\text {label }}(L)=E_{\text {data }}(L)+E_{\text {smooth }}(L)
$$

The energy function is defined by a data term and a smoothness term. The data term $E_{\text {data }}(L)$ is used to explain the data by labelling the cells. The smoothness term $E_{\text {smooth }}(L)$ represents a pairwise cost for adjacent cells.

Data term. $E_{\text {data }}$ consists of a sum of unary functions $D_{c}\left(l_{c}\right)$, each of which denotes the cost of assigning a label $l_{c} \in\left\{l_{1}, \ldots, l_{\text {Nroom }}, l_{\text {out }}\right\}$ to a cell $c_{i} \in\left\{c_{1}, \ldots, c_{n}\right\}$ :

$$
E_{\text {data }}(L)=\sum_{c \in c\{1, \ldots, n\}} D_{c}\left(l_{c}\right)
$$

The $D_{c}\left(l_{c}\right)$ value is low if the cell is likely to belong to $l_{c}$. All point clouds of segmented rooms are projected onto the horizontal plane to determine cell labels according to the point cloud room labels $\left\{l_{1}, \ldots, l_{\text {Nrooms }}\right\}$. A cell is considered as the outside world $l_{\text {out }}$ if it is not represented by any scanning points. The unary cost is then defined as:

$$
D_{c}\left(l_{c}\right)=\alpha \cdot \operatorname{area}(s) \cdot\left\|V(s)-I_{c}\right\|_{1}
$$

Where $V(s)$ is the label vector of cell $s, I_{c}$ is the ideal label vector of cell $s$ for label $l_{c}$, area $(s)$ is the cell's area, the parameter $\alpha$ is a weighting factor to define the importance of the data term.

Smoothness term. $E_{\text {smooth }}(L)$ is used to regularize the label by penalizing the assignment of different labels to adjacent cells, and consists of a sum of binary functions $R_{c, d}\left(l_{c}, l_{d}\right)$, each of which denotes the cost of assigning two different labels to adjacent cells. The binary function is defined as:

$$
E_{\text {smooth }}(L)=\sum_{(c, d) \in c\{1, \ldots, n\}} R_{c, d}\left(l_{c}, l_{d}\right)
$$

The $E_{\text {smooth }}(L)$ should be low if the segment of the wall represented by edge $e$ are supported by wall points with two different labels $l_{c}, l_{d}$. The label vector of the edge $e$ is determined according to the room labels of projected points on wall surfaces. The binary cost is defined as:

$R_{c, d}\left(l_{c}, l_{d}\right)=\left\{\begin{array}{l}\beta \cdot \operatorname{len}(e) \cdot\left(\left\|V(e)-I_{c d}\right\|_{1}+\gamma V_{0}(e)\right),\left(l_{c} \neq l_{d}\right) \\ 0,\left(l_{c}=l_{d}\right)\end{array}\right.$

Where $c, d$ are the cells separated by edge $e, \operatorname{len}(e)$ is 
the edge length, $\beta, \gamma$ are weight parameters, $V(e)$ is the label vector of edge $e, I_{c d}$ is the ideal label vector for label $l_{c, d}$. The additional term $V_{0}(e)$ will penalize the edges of which both labels on each side are $l_{\text {out }}$. Finally, the energy function can be minimized using multi-label graph cuts [48] to label the cells as different rooms or the exterior. Once the labels of cells are determined, room structures can be reconstructed. The wall of each room is the centerline that determined by the average location of the point clouds with labels $l_{c}, l_{d}$, and the heights of the floor and ceiling are estimated by the points of on horizontal surfaces (ceiling and floor). The reconstructed surface models of different rooms are shown in Fig. 7 (c). The thickness of room models is given as a fixed value $m^{T}$. The walls with thickness are generated by extruding half of the thickness along both sides of the centerline. The floors and ceilings are generated by extruding a thickness along the normal direction towards the outside of the room. The final volumetric solid models of rooms are shown in Fig. 7 (d).

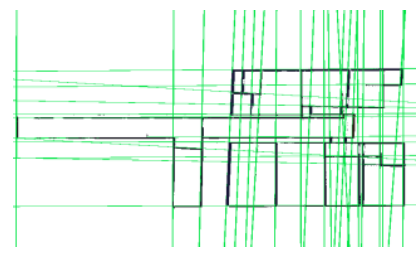

(a)Segmented walls fitted with linear (b) primitives

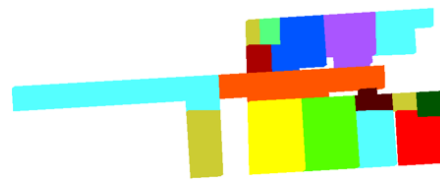

(c) Surface models of different rooms (d) Volumetric solid models of rooms Fig. 7. Overview of model reconstruction

\section{EXPERIMENT}

\section{A. Test Data}

Three ISPRS benchmark datasets acquired by MLS were tested, as illustrated in Fig. 8. The specifications of the sensors and point clouds are shown in Table I. The first dataset (TUB2) is captured in one of the buildings at Technical University of Braunschweig, Germany, using a handheld scanner, Zeb-Revo. The data were acquired across two floors connected by a staircase. The point clouds and trajectories are shown as Fig. 8 (a). The first floor of the building contains 14 rooms, 2 corridors, 8 windows, and 23 doors, some of which are open whereas others are closed. The second floor includes 7 rooms, 2 corridors, 13 windows and 28 doors [49]. The walls have different thicknesses and the ceilings have different heights. The second dataset (UVigo) is captured at University of Vigo, Spain, using a backpack-based mobile mapping system, which provides both the point clouds and the trajectories, as shown in Fig. 8 (b). The scene includes one room, an entrance hall, one curtain wall, 20 windows, 7 simple doors and several circular columns in the middle [49]. The level of noise in the data is moderate. The third dataset (UoM) is acquired in the block B of the engineer building at University of Melbourne, Australia, using a handheld scanner, Zeb1. The scene includes 7 rooms and 14 doors (open or closed), with walls of different thickness and blinded windows, as shown in Fig. 8 (c).

TABLE I

SPECIFICATIONS OF TESTED SENSORS AND POINT CLOUDS

\begin{tabular}{|c|c|c|c|}
\hline \multicolumn{4}{|c|}{ Technical specifications of the sensors } \\
\hline Sensor & $\begin{array}{c}\text { ZEB } \\
\text { REVO }\end{array}$ & UVigo & ZEB1 \\
\hline Max range & $30 \mathrm{~m}$ & $100 \mathrm{~m}$ & $20 \mathrm{~m}$ \\
\hline Speed (points/sec) & $43 * 103$ & $300 * 103$ & $43 * 103$ \\
\hline $\begin{array}{c}\text { Horizontal Angular } \\
\text { Resolution }\end{array}$ & $0.625^{\circ}$ & $0.1-0.4^{\circ}$ & $0.25^{\circ}$ \\
\hline $\begin{array}{c}\text { Vertical Angular } \\
\text { Resolution }\end{array}$ & $1.8^{\circ}$ & $2.0^{\circ}$ & $3.5^{\circ}$ \\
\hline Angular FOV & $\begin{array}{l}270^{*} \\
360^{\circ}\end{array}$ & $30 * 360^{\circ}$ & $270^{*} 150^{\circ}$ \\
\hline Relative Accuracy & $2-3 \mathrm{~cm}$ & $3 \mathrm{~cm}$ & $2-3 \mathrm{~cm}$ \\
\hline Absolute Accuracy & $3-30 \mathrm{~cm}$ & -- & -- \\
\hline Number of points & $\begin{array}{c}11628 \\
186\end{array}$ & $\begin{array}{c}89787 \\
72\end{array}$ & $\begin{array}{c}13422 \\
135\end{array}$ \\
\hline $\begin{array}{c}\text { Mean point spacing } \\
(\mathrm{m})\end{array}$ & 0.008 & 0.01 & 0.007 \\
\hline Trajectory & 65889 & 1108 & No \\
\hline Timestamps & Yes & Yes & No \\
\hline Color & No & No & No \\
\hline $\begin{array}{l}\text { The range of } \mathrm{x}-, \mathrm{y}-\text {, } \\
\mathrm{z} \text {-axis }\end{array}$ & $\begin{array}{c}23.72, \\
43.23, \\
11.40\end{array}$ & $\begin{array}{c}27.31 \\
27.2,3.69\end{array}$ & $\begin{array}{c}27.05 \\
18.74,3.26\end{array}$ \\
\hline
\end{tabular}

(a) Point clouds and trajectories acquired by HLS, ZEB-REVO.

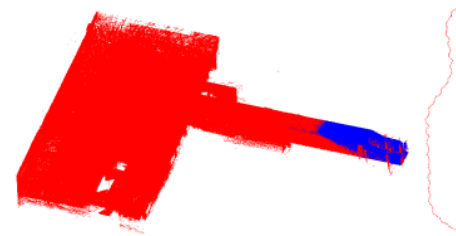

(b) Point clouds and trajectories acquired by BLS, UVigo

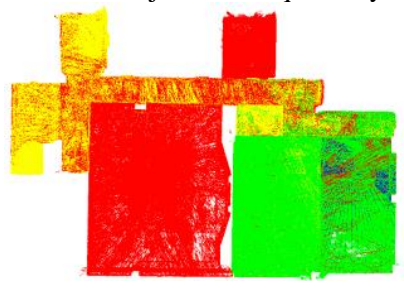

(c) Point clouds acquired by a handheld scanner, ZEB 1

Fig. 8. The experiment data

\section{B. Parameter Study}

The parameters of proposed indoor reconstruction method for three datasets are shown in Table II. Experiments show that most of the parameters are insensitive to different point clouds 
in various indoor scenes and only a few parameters need manual modification. $p_{n}$ is the parameter used in the RANSAC plane segmentation, where planes with supporting points less than this threshold will be discarded. $\operatorname{bin}, p_{v}, p_{d}$ are three parameters used to extract horizontal planes. The choice of histogram bin size is determined by data point density, and $p_{v}, p_{d}$ are determined according to the indoor scene and used to identify the location of the main horizontal plane. $p_{n}, p_{v}, p_{d}$ depend on different indoor scenes according to our experiments hence need to be adjusted. For extracting vertical walls, $n_{z}, s_{x y}$ are the parameters utilized in the vertical plane refinement. Meanwhile, $\theta_{t}, d_{t}$ are set to merge planar segments that represent the same wall. For door detection, the point clouds of door location are transformed into cells, where $C_{s}$ denotes the size of a cell, and $r_{t}$ is the parameter utilized in Hough transform to avoid interference from outliers and noise. $\theta_{a}, d_{c}$ are used to merge line segments that represent the same door. $l_{d}, h_{d}$ denote the length and height of the regularized doors. Extraction of windows is similar to that of doors, $l_{w}, h_{w}$ are the length and height of windows. For room segmentation, $R, N$ are searching radius and point number thresholds in visibility analysis. $t_{\text {adapted }}$ denotes the adaptive threshold value based on the OTSU method to obtain trajectory groups. $M_{\text {thread }}$ is the threshold value used to merge trajectories. For structural model reconstruction, $\alpha, \beta, \gamma$ are weight parameters used for balancing different terms in the multi-label graph cuts method. $m_{T}$ is the thickness of reconstruction model interpreted from the real scene.
TABLE. II.

PARMETER OF THE PROPOSED INDOOR RECONSTRUCTION

\begin{tabular}{|c|c|c|}
\hline Parameters & Values & Descriptions \\
\hline \multicolumn{3}{|c|}{ Plane segmentation by RANSAC } \\
\hline$p_{n}$ & $5000 *$ & $\begin{array}{l}\text { The minimum number of } \\
\text { supporting points } \\
\text { in a plane }\end{array}$ \\
\hline \multicolumn{3}{|c|}{ Extracting horizontal planes } \\
\hline bin & $0.03 \mathrm{~m}$ & $\begin{array}{l}\text { Bin size of } \mathrm{x}-, \mathrm{y}-, \mathrm{z}-\text { coordinate } \\
\text { histogram }\end{array}$ \\
\hline$p_{v}$ & $330000 *$ & $\begin{array}{l}\text { The threshold value of peak } \\
\text { extraction }\end{array}$ \\
\hline$p_{d}$ & $0.2 \mathrm{~m}^{*}$ & $\begin{array}{l}\text { The minimum distance between } \\
\text { adjacent peaks }\end{array}$ \\
\hline \multicolumn{3}{|c|}{ Regular vertical walls } \\
\hline$n_{z}$ & 0.087 & $\begin{array}{l}\text { The threshold of normal } \\
\text { component of the } \mathrm{z} \text { axis }\end{array}$ \\
\hline$s_{x y}$ & 0.25 & $\begin{array}{l}\text { The slope threshold of extracted } \\
\text { planes }\end{array}$ \\
\hline$\theta_{t} / d_{t}$ & $18 \% / 0.1 \mathrm{~m}$ & $\begin{array}{l}\text { The angle threshold and distance } \\
\text { threshold or merging planes }\end{array}$ \\
\hline \multicolumn{3}{|r|}{ Extracting doors } \\
\hline$C_{s}$ & $0.3 \mathrm{~m}$ & The size of the cell \\
\hline$r_{t}$ & $\pi / 360$ & $\begin{array}{l}\text { The radian threshold of extracted } \\
\text { line by Hough transform }\end{array}$ \\
\hline$\theta_{a} / d_{c}$ & $10^{\circ} / 0.2 \mathrm{~m}$ & $\begin{array}{l}\text { The angle threshold and distance } \\
\text { threshold for merging line } \\
\text { segments }\end{array}$ \\
\hline$l_{d} / h_{d}$ & $1 \mathrm{~m} / 2.2 \mathrm{~m}$ & $\begin{array}{l}\text { The length and height of } \\
\text { regularized door }\end{array}$ \\
\hline \multicolumn{3}{|c|}{ Extracting windows } \\
\hline$l_{w} / h_{w}$ & $1 \mathrm{~m} / 1 \mathrm{~m}$ & $\begin{array}{l}\text { The length and height of } \\
\text { regularized window }\end{array}$ \\
\hline \multicolumn{3}{|c|}{ Segmentation of rooms } \\
\hline$R / N$ & $0.1 \mathrm{~m} / 5$ & $\begin{array}{l}\text { The searching radius and point } \\
\text { number thresholds in visibility } \\
\text { analysis for determining the doors } \\
\text { near trajectories }\end{array}$ \\
\hline$t_{\text {adapted }}$ & * & $\begin{array}{l}\text { The adaptive threshold value of } \\
\text { obtaining trajectory groups }\end{array}$ \\
\hline$M_{\text {thread }}$ & 0.6 & $\begin{array}{l}\text { The threshold value of merging } \\
\text { trajectories }\end{array}$ \\
\hline \multicolumn{3}{|c|}{ Structural model reconstruction } \\
\hline$\alpha, \beta, \gamma$ & $\begin{array}{l}1.0,1.0,0 . \\
1\end{array}$ & $\begin{array}{l}\text { The weight parameters of } \\
\text { multi-label graph cuts }\end{array}$ \\
\hline$m_{T}$ & $0.22 \mathrm{~m}$ & $\begin{array}{l}\text { The wall thickness of } \\
\text { reconstructed model }\end{array}$ \\
\hline
\end{tabular}

(The asterisk '*' represents the parameters determined by the datasets)

\section{Results}

The proposed algorithm was written in $\mathrm{C}++$, edited by Microsoft Visual Studio 2017, and is performed on a Window 10 64-bit operating system with an Alienware Intel (R) Core (TM) i7-7700HQ CPU @ $2.80 \mathrm{GHz}$ and a 16GB RAM.

For the first data, the extracted ceilings, floors and walls are shown in Figs. 9 (a) and (b). The openings (doors and windows) 
of the first and the second floors are shown in Fig. 9 (c), in which the red ones are windows, and the green ones are doors, but the purple ones are windows falsely detected as doors. Accurate structural elements are important for room segmentation and structure vector modelling. The room segmentation results of the first and second floors are shown in Fig. 9 (d), from which it can be observed that the whole point clouds are correctly subdivided into different rooms. Fig. 9 (e) shows the extruded volumetric solid models of ceilings, floors and walls of the first floor. Fig 9. (f) shows the extruded volumetric solid models of the first floor, second floor and whole building. The lower height of each ceiling is determined by the average height of the point clouds of on the ceiling surface. The upper height of the floor is the average height of the point clouds on the floor surface. Each wall is given a thickness and a centerline, which is determined by the average location of the point clouds on the wall surfaces. The whole model is given a fixed thickness $t=0.22 m$. However, the actual walls may have different thickness. Finally, the surface models of segmented rooms (without extrusions) are shown in different colors in Fig. $9(\mathrm{~g})$. For the second data, the whole room volumetric solid model with thickness is shown as the Fig. 10 (a), which includes one room, an entrance hall, one curtain wall. Figs. 10 (b) and (c) show the detailed volumetric solid models of walls and cylinders. For the third data, Fig. 11 shows all the room segments and volumetric solid and surface models of a whole floor.

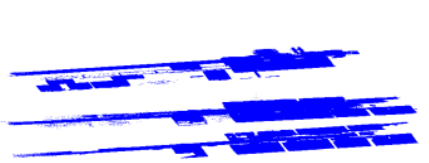

(a) The detected ceilings and floors

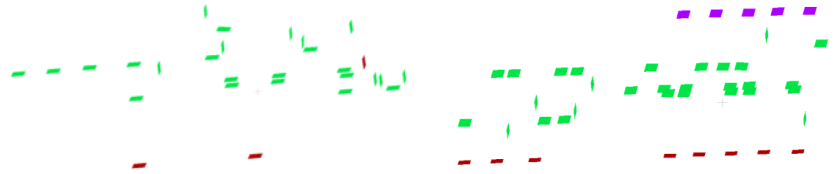

(c) Doors (green) and windows (red and purple) of the first and second floor

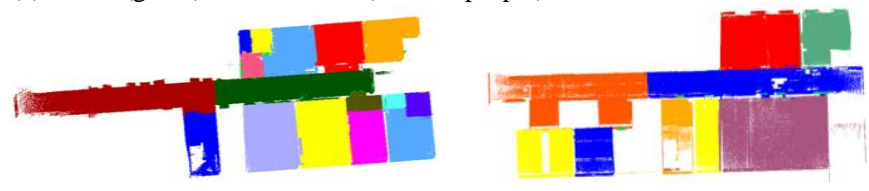

(d) Segmented rooms of the first and second floor
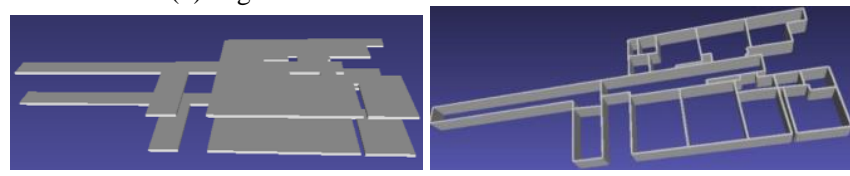

(e) Volumetric solid models of the ceiling, floor and walls of the first floor

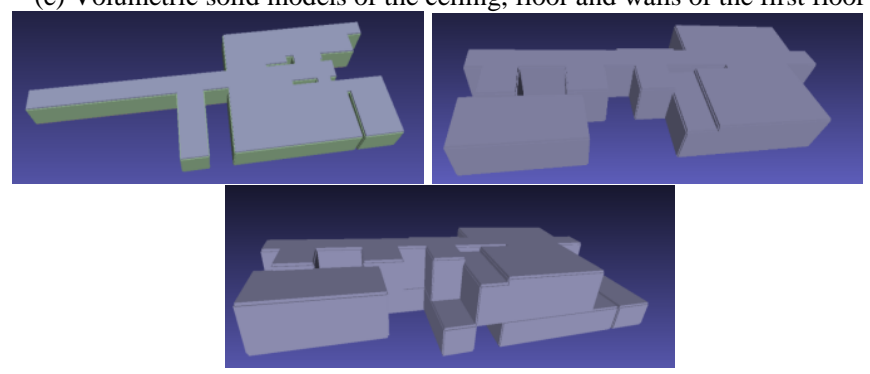

(f) Volumetric solid models of the first and second floor

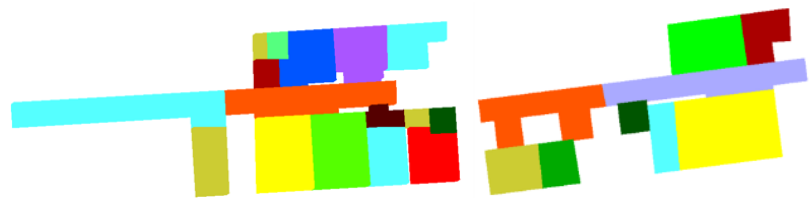

(g) Surface models of segmented rooms on the first and second floor Fig. 9. Element extraction, segmented rooms, volumetric solid and surface model results from the handheld scanning data

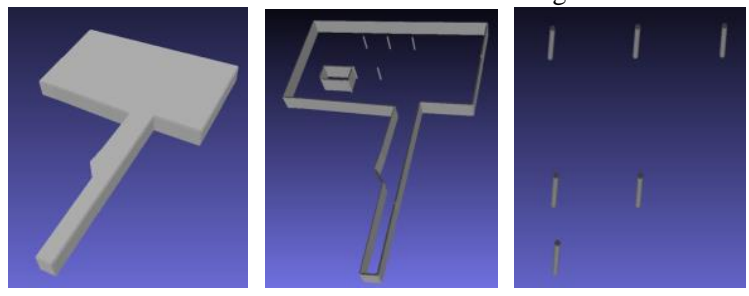

(a) The model of the room (b) Vector model of the wall (c) Cylindrical models Fig. 10. Volumetric solid model results of the backpack-based mobile scanning data
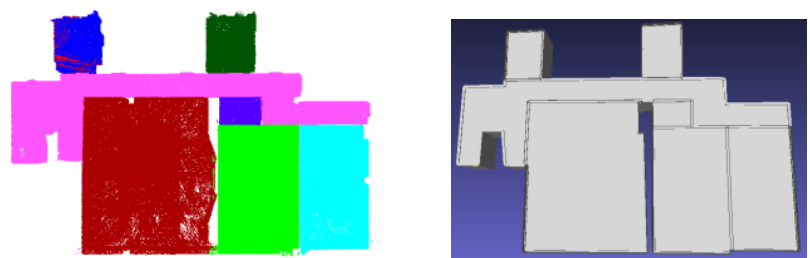

(a) Room segments

(b) Volumetric solid models of the whole floor
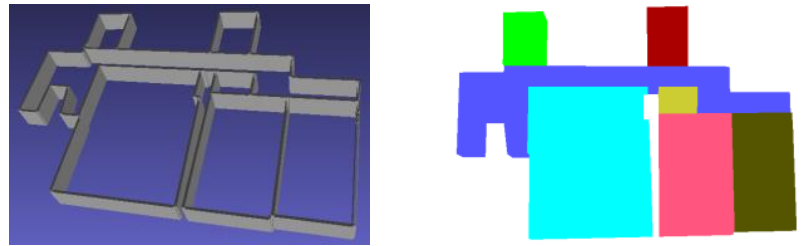

(c) Volumetric solid models of a whole floor (d) Surface models of room segments

Fig. 11. Volumetric solid and surface model results of the backpack-based mobile scanning data

\section{EVALUATION AND DISCUSSION}

The proposed approach was tested on three datasets that captured by MLS. Both quantitative and qualitative evaluations are carried out to assess the geometric elements, semantics and topological relations of the reconstructed indoor models. The qualitative evaluation mainly checks the presence and correctness of the semantic attribute as well as the spatial and topological relations. The completeness, correctness and accuracy of the reconstructed elements are quantitatively evaluated through a comparison between the reference model $R$ and the automatically reconstructed model referred to as the source model $S$ [49]. The completeness is defined as:

$$
M_{\text {Comp }}(b)=\frac{\sum_{i=1}^{n} \sum_{j=1}^{m}\left|S^{i} \cap b\left(R^{j}\right)\right|}{\sum_{j=1}^{m}\left|R^{j}\right|}
$$

Where the intersection areas are computed over all surfaces of $S^{i}$ and $R^{j}$, the completeness value varies with the size of the buffer $b$ [44] .

The correctness is defined as: 


$$
M_{\text {Corr }}(b)=\frac{\sum_{i=1}^{n} \sum_{j=1}^{m}\left|S^{i} \cap b\left(R^{j}\right)\right|}{\sum_{i=1}^{n}\left|S^{i}\right|}
$$

The accuracy is defined as:

$$
M_{A c c}(r)=\operatorname{Med}\left\|\pi_{j}^{T} p_{i}\right\|, \text { if }\left\|\pi_{j}^{T} p_{i}\right\| \leq r
$$

Where $\left\|\pi_{j}^{T} p_{i}\right\|$ is the perpendicular distance between a vertex point $P_{i}$ in the source model and the plane $\pi_{j}$ in the reference model, and $r$ is the cut-off value to avoid the influence of incompleteness or incorrectness of the source model.

The quantitative evaluation results of structural elements are given in Table III.

TABLE. III.

DATASETS AND RESULTS IN OUR EXPERIMENTS

\begin{tabular}{|c|c|c|c|c|}
\hline Data & $\begin{array}{c}\text { First } \\
\text { Data } \\
\text { Floor 1 }\end{array}$ & $\begin{array}{c}\text { First } \\
\text { Data } \\
\text { Floor 2 }\end{array}$ & $\begin{array}{c}\text { Second } \\
\text { Data }\end{array}$ & Third Data \\
\hline $\begin{array}{c}\text { Number } \\
\text { of points }\end{array}$ & 6623311 & 5004875 & 8978772 & 13414298 \\
\hline $\begin{array}{c}\text { Processing } \\
\text { time (s) }\end{array}$ & 224 & 322 & 65 & 97 \\
\hline $\begin{array}{c}\text { Actual/ } \\
\text { detected } \\
\text { rooms } \\
\text { corridors) }\end{array}$ & $14(2) /$ & $7(2) /$ & $1(1) /$ & $7 / 7$ \\
\hline $\begin{array}{c}\text { Actual/ } \\
\text { detected } \\
\text { doors }\end{array}$ & $23 / 22$ & $28 / 29$ & $7 / 4$ & $14 / 2$ \\
\hline $\begin{array}{c}\text { Actual/ } \\
\text { detected } \\
\text { windows }\end{array}$ & $8 / 3$ & $13 / 8$ & $20 / 0$ & $0 / 0$ \\
\hline $\begin{array}{c}\text { Actual/ } \\
\text { detected } \\
\text { cylinders }\end{array}$ & $0 / 0$ & $0 / 0$ & $6 / 6$ & $0 / 0$ \\
\hline
\end{tabular}

\section{A. HLS Benchmark (first)Dataset Evaluation}

The first data is the Benchmark data that collected by a handheld scanner. The structural elements were extracted accurately, such as ceilings, floors, walls, doors and windows. The structural elements of the first floor are shown in Fig. 12 (a). 22 openings are detected as doors, 18 of which are correct. 4 pink circles are false detection, and 5 red circles are miss detections. The method cannot detect the 5th door in red circle as it is closed, and the failure of other four doors are due to the sparseness of the point clouds. There are only 3 windows extracted, mostly because the noise level is high near glass windows. There are 14 rooms and 2 corridors that are all correctly segmented, as shown in Fig. 9 (d) (left). The structural elements of the second floor are shown in Fig. 12 (b). 29 openings are extracted as doors, 24 of which are correct, but 5 pink openings are windows that falsely detected as doors. The door in yellow circle is not detected because it is closed, and the double door in the green circle is extracted as single door because one side is closed during data acquisition. There are 8 windows correctly detected. There are 7 rooms and 2 corridors which are correctly segmented, as shown in Fig. 9 (d) (right).

The qualitative evaluations through visual inspections show that the openings are correctly positioned and completely embedded in the walls, as shown in Figs. 12 (a) and (b). The input point clouds and output models are well matched, as shown in Fig. 12 (c). The 3D reconstructed models were quantitatively evaluated based on the visual surface model in terms of completeness, correctness and accuracies [5]. For the whole building, Figs. 12(d)-(h) present the completeness and correctness and accuracies of planar source models plotted against the reference model with a buffer size at $10 \mathrm{~cm}$ and the cut-off distance ranging from $1 \mathrm{~cm}$ to $15 \mathrm{~cm}$, respectively. The curves show that the increase of buffer size will improve the completeness and correctness rates, in contrast, the accuracy decreases with the larger cut-off values. For the first floor, Fig. 12 (d) shows that floors and ceilings achieve high completeness $(61 \%)$ and correctness $(86 \%)$ at small buffer sizes. However, the walls of surface models reach the completeness (43\%) and correctness $(61 \%)$ at the buffer size of $10 \mathrm{~cm}$. The accuracy $(7.5 \mathrm{~cm})$ of walls of all surface models is lower than those of floors and ceilings $(2 \mathrm{~cm})$ ranging from $1 \mathrm{~cm}$ to $15 \mathrm{~cm}$, as shown in Fig. 12 (h). The reason is that the centerline of wall model is determined by the average location of the point clouds on the inner and outer wall surfaces. However, the reference model is based on the visual surface model where the interior wall has two sides. Hence, the accuracy of $7.5 \mathrm{~cm}$ is closed to a half of real thickness. Surfaces of the floor and ceiling are estimated by the points of on horizontal surfaces (ceiling and floor). Figs. 12 (d) and (h) illustrates that the doors and windows of the models achieve a low completeness (18\%) and correctness (20\%) with accuracy $(6.5 \mathrm{~cm})$. The reason is that the point clouds near the windows and the incompleteness of wall elements have random noise. Fig. 12 (f) shows that completeness and correctness of the surface model for the second floor, which are much higher than the first floor. As illustrated in Figs. 12 (e) and (g), it can be verified by visual inspection that the completeness and correctness of most of surface models are more than $60 \%$ and only a few are less than $30 \%$ for the first and second floors. Moreover, the surface models have higher correctness than completeness. Figs. 12 (h) shows that the average accuracy of walls, floors, ceilings, doors and windows of whole building is close to $5 \mathrm{~cm}$.

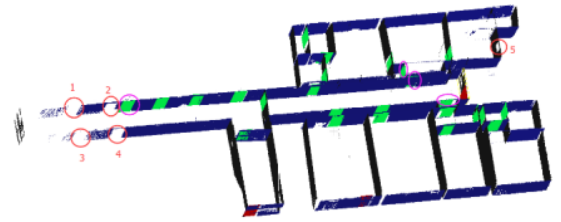

(a) The doors (green) and windows (red) combined with detected wall points on the first floor

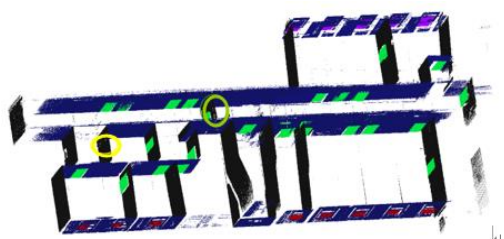

(b) The doors (green) and windows (red, purple) combined with detected wall points on the Second floor 


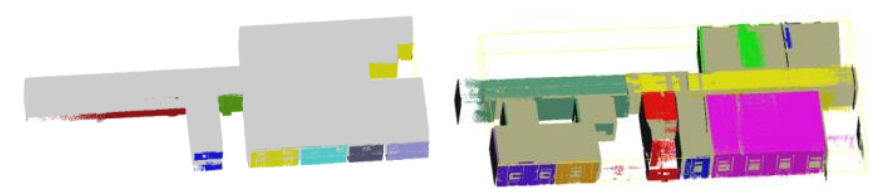

(c) Matching between the point clouds of segmented rooms and the reconstructed model on the first and second floors Completeness Of Walls\&Floors_Ceilings\&Doors_Windows

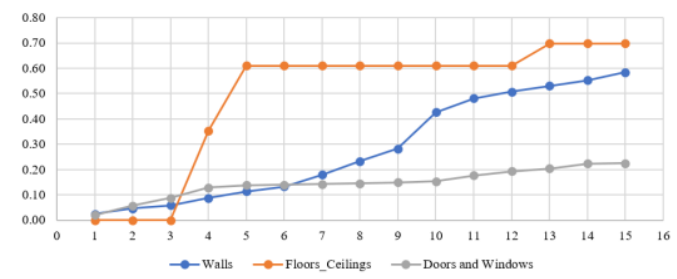

Correctness Of Walls\&Floors_Ceilings\&Doors_Windows

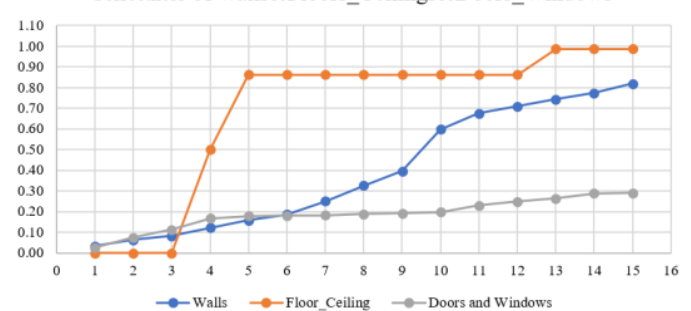

(d) Completeness and correctness of surface models (walls, floors, ceilings, doors and windows) for the first floor

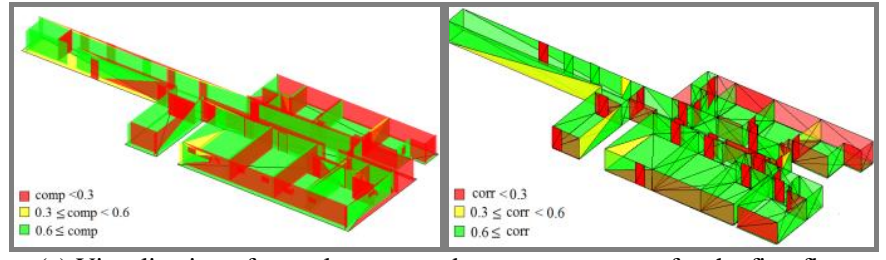

(e) Visualization of completeness and correctness errors for the first floor measured with buffer size at $10 \mathrm{~cm}$

Completeness Of Walls\&Floors_Ceilings\&Doors_Windows

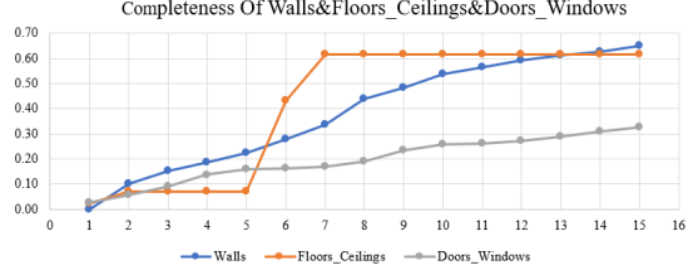

Correctness Of Walls\&Floors_Ceilings\&Doors_Windows

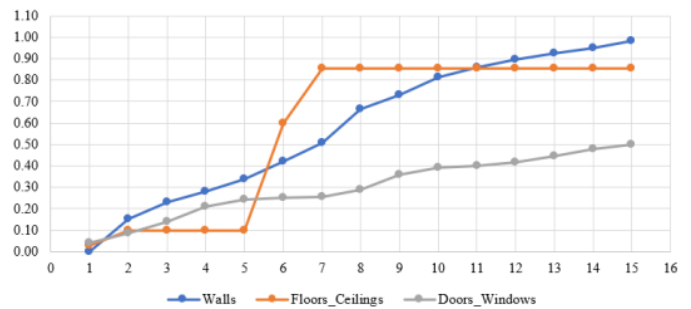

(f) Completeness and correctness of surface model for the second floor

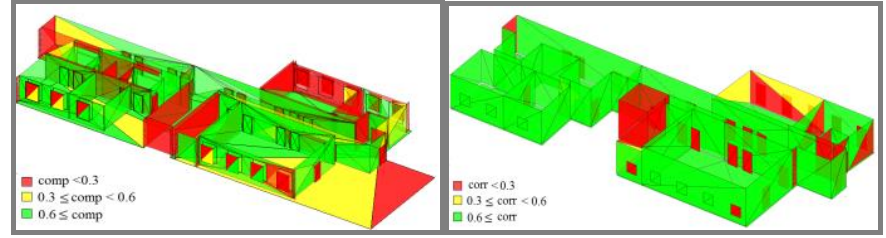

(g) Visualization of completeness and correctness errors for the second floor

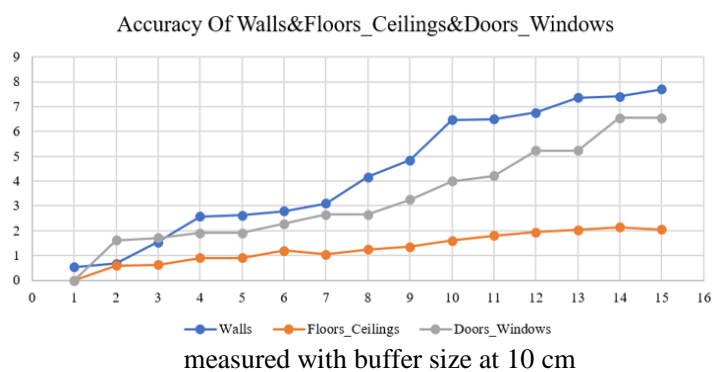

(h) The accuracy of surface model for the whole building

Fig. 12 Results of the qualitative and quantitative evaluation of reconstructed surface models for the HLS (first) dataset. (a-c) are the qualitative evaluation results. (d-h) are the quantitative evaluation results

\section{B. BLS (second) and HLS (third) Dataset Evaluation}

The structural element extraction and 3D model reconstruction from the second, third datasets were also qualitatively and quantitatively evaluated. For the second data that have high level of noise and sparsity, the qualitative results are shown in Figs. 13 (a) and (b), from which it can be observed that the extracted doors, cylinders, reconstructed models and the point clouds are well matched. Fig. 13 (c) shows some examples of openings from the original data. The yellow rectangles indicate doors that have no enough point above, and the red rectangle shows a series of glass windows that have noise points in the openings. Therefore, there were only 4 doors extracted during opening detection. The quantitative results of the structural element extraction are showed in Table III.

For the third data, the results are shown in Figs. 13 (d) and (e), in which the extracted doors, walls, and the final reconstructed model are well matched with the point clouds. Fig. 13 (d) shows that only two doors (green) are detected as other doors are closed, and all windows are not captured in the original data. The quantitative results of the structural element extraction are showed in Table III.

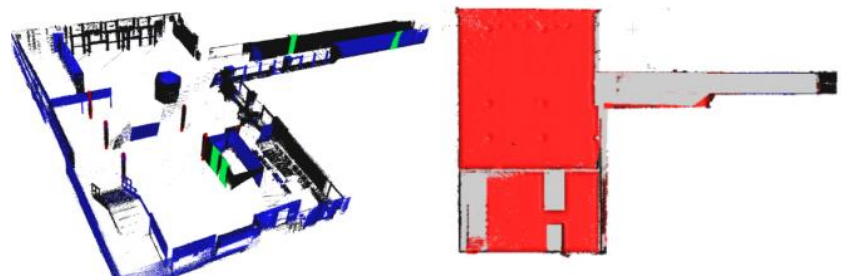

(a) The doors (green) and cylinders (red) (b) Matching between the whole point combined with the point clouds clouds and the reconstructed model

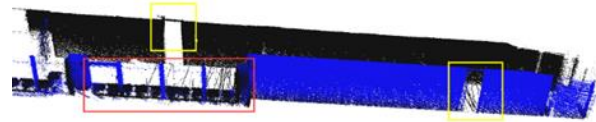

(c) The location of doors (yellow rectangle) and windows (red rectangle) in the point clouds

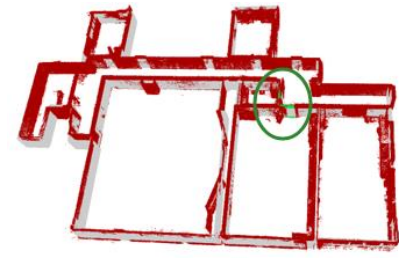

(d) The doors (green), walls (gray) combined with the point clouds

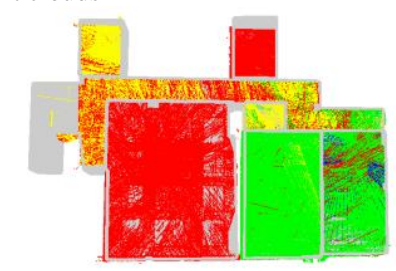

(e) Matching between the whole point cloud and the reconstructed model
evaluations of the BLS and HLS data 


\section{Limitations}

This paper proposed a method to reconstruct the indoor environment that can have multi-floors, multi-rooms, and complicated connections from mobile laser scanning point clouds. However, there are few limitations. For example, it is only suitable for modelling indoor environments with Manhattan-world structures so it may have difficulties in more complex scenes. For window and door detections, the method only relies on the geometric and semantic information of the point clouds. The noise from the nearby openings may cause false detections. For structural modelling, serious data incompleteness will affect the modelling results. Lastly, the reconstructed vector elements are given a fixed thickness, which can be different in the real structures.

\section{CONCLUSION AND OUTLOOK}

3D reconstruction of indoor environment from laser scanning point clouds has attracted many attentions from the field of computer graphics, computer vision and photogrammetry [50] for the past ten years. But it is still a challenging task to automatically model complex indoor environment. This paper proposed an automatic method to address the structural modelling of indoor environment with multiple floors, multiple rooms, and complicated connections based on 3D point clouds. The method consists of three main steps with little or no manual interaction: the extraction of structural elements such as the ceilings, floors, walls, doors, windows and cylinders; the segmentation of room based on the visibility analysis of trajectories and constraints of structured elements; and the reconstruction of volumetric solid and surface models based on multi-label graph cuts.

The input MLS data are both point clouds and trajectories of the whole scene, different from most previous studies. The results of geometric elements, semantic and topological relations of the reconstructed models are qualitatively and quantitatively evaluated. The assessment of the HLS data from ISPRS shows that the completeness and correctness of reconstructed surface models are mostly more than $60 \%$. The average accuracy of model is close to $5 \mathrm{~cm}$. Experiments showed that promising results can be obtained from the proposed method, even the walls are modelled in the center rather on both sides as in the reference. These are two different modelling strategies. The comparison between them will be investigated in future work. However, the method can be improved at least in two aspects in the future. The combination of point clouds with images to improve the opening detection accuracy is planned. Meanwhile, the reconstructed models can be textured by images for augmented reality applications.

\section{ACKNOWLEDGMENT}

We would like to thank the ISPRS Commission WG IV/5 for provision of the data and the evaluation of the results.

\section{REFERENCES}

[1] A.A. Diakité, and S. Zlatanova, "Valid space description in BIM for 3D indoor navigation," International Journal of 3-D Information Modeling (IJ3DIM), vol. 5, no. 3, pp. 1-17, 2016.

[2] T. Cerovsek, "A review and outlook for a "Building Information Model'(BIM): A multi-standpoint framework for technological development," Advanced engineering informatics, vol. 25, no. 2, pp. 224-244, 2011.

[3] E. Turner, P. Cheng, and A. Zakhor, "Fast, automated, scalable generation of textured 3d models of indoor environments," IEEE Journal of Selected Topics in Signal Processing, vol. 9, no. 3, pp. 409-421, 2015.

[4] S. Zlatanova, L. Liu, G. Sithole, J.Q. Zhao, and F. Mortari, "Space subdivision for indoor applications," GISt Report No. 66, 2014.

[5] K. Khoshelham, H. Tran, L. Díaz-Vilariño, M. Peter, Z. Kang, and D. Acharya, "An Evaluation Framework for Benchmarking Indoor Modelling Methods," ISPRS - International Archives of the Photogrammetry, Remote Sensing and Spatial Information Sciences, vol. 42, no. 4, 2018.

[6] C. Wang, S.W. Hou, C.L. Wen, Z. Gong, Q. Li, X.T. Sun, and J. Li, "Semantic line framework-based indoor building modeling using backpacked laser scanning point cloud," Isprs Journal of Photogrammetry and Remote Sensing, vol. 143, pp. 150-166, 2018.

[7] J. Wang, K. Xu, L.G. Liu, J.J. Cao, S.J. Liu, Z.Y. Yu, and X.D. Gu, "Consolidation of low-quality point clouds from outdoor scenes," in Proceedings of the Eleventh Eurographics/ACMSIGGRAPH Symposium on Geometry Processing, Eurographics Association, 2013, pp. 207-216.

[8] L. Díaz-Vilariño, P. Boguslawski, K. Khoshelham, H. Lorenzo, and L. Mahdjoubi, "Indoor navigation from point clouds: 3D modelling and obstacle detection," ISPRS - International Archives of the Photogrammetry, Remote Sensing and Spatial Information Sciences XLI-B4, 2016, pp. 275-281.

[9] I. Armeni, O. Sener, A.R. Zamir, H. Jiang, I. Brilakis, M. Fischer, and S. Savarese, "3d semantic parsing of large-scale indoor spaces," in Proceedings of the IEEE Conference on Computer Vision and Pattern Recognition, 2016, pp. 1534-1543.

[10] B. Xiong, M. Jancosek, S.O. Elberink, and G. Vosselman, "Flexible building primitives for 3D building modeling," ISPRS Journal of Photogrammetry and Remote Sensing, vol. 101, pp. 275-290, 2015.

[11] A. Monszpart, N. Mellado, G.J. Brostow, and N.J. Mitra, "RAPter: rebuilding man-made scenes with regular arrangements of planes," ACM Transactions on Graphics, vol. 34, no. 4, pp. 103:1-103:12, 2015.

[12] S. Ochmann, R. Vock, R. Wessel, and R. Klein, "Automatic reconstruction of parametric building models from indoor point clouds," Computers \& Graphics, vol. 54, pp. 94-103, 2016.

[13] C. Mura, O. Mattausch, and R. Pajarola, "Piecewise - planar Reconstruction of Multi- room Interiors with Arbitrary Wall Arrangements," Computer Graphics Forum, 2016, vol. 35, no.7, pp. 179-188.

[14] Y. Zheng, M. Peter, R. Zhong, S. Oude Elberink, and Q. Zhou, "Space subdivision in indoor mobile laser scanning point clouds based on scanline analysis," Sensors, vol. 18, no. 6, pp. 1838, 2018.

[15] M. Previtali, L. Díaz-Vilariño, and M. Scaioni, "Towards automatic reconstruction of indoor scenes from incomplete point clouds: door and window detection and regularization," ISPRS TC-4 Mid-term Symposiumm 2018, 2018, vol. 42, no. 4, pp. 507-514.

[16] L. Li, F. Su, F. Yang, H.H. Zhu, D.L. Li, X.K. Zuo, F. Li, Y. Liu, and S. Ying, "Reconstruction of Three-Dimensional (3D) Indoor Interiors with Multiple Stories via Comprehensive Segmentation," Remote Sensing, vol. 10, no. 8, pp. 1281, 2018.

[17] R.S. Wang, L. Xie, and D. Chen, "Modeling indoor spaces using decomposition and reconstruction of structural elements," Photogrammetric Engineering \& Remote Sensing, vol. 83, no. 12, pp. 827-841, 2017.

[18] L. Díaz-Vilariño, E. Verbree, S. Zlatanova, and A. Diakité, "Indoor modeling from slam-based laser scanner: door detection to envelope reconstruction," International Archives of the Photogrammetry, Remote Sensing \& Spatial Information Sciences, vol. 42, 2017.

[19] E. Turner, and A. Zakhor, "Floor plan generation and room labeling of indoor environments from laser range data," 2014 International Conference on Computer Graphics Theory and Applications (GRAPP), IEEE, 2014, pp. $1-12$.

[20] T. Hackel, J.D. Wegner, and K. Schindler, "Fast semantic segmentation of 3D point clouds with strongly varying density," ISPRS Annals of Photogrammetry, Remote Sensing \& Spatial Information Sciences, vol. 3, no. 3, 2016. 
[21] M. Weinmann, B. Jutzi, S. Hinz, and C. Mallet, "Semantic point cloud interpretation based on optimal neighborhoods, relevant features and efficient classifiers," ISPRS Journal of Photogrammetry and Remote Sensing, vol. 105, pp. 286-304, 2015.

[22] H. Thomas, F. Goulette, J.-E. Deschaud, and B. Marcotegui, "Semantic Classification of 3D Point Clouds with Multiscale Spherical Neighborhoods," 2018 International Conference on 3D Vision (3DV), 2018, pp. 390-398.

[23] I. Tsochantaridis, T. Hofmann, T. Joachims, and Y. Altun, "Support vector machine learning for interdependent and structured output spaces," in Proceedings of the twenty-first international conference on Machine learning, ACM, 2004, pp. 104.

[24] A. Rottmann, Ó.M. Mozos, C. Stachniss, and W. Burgard, "Semantic place classification of indoor environments with mobile robots using boosting," AAAI, 2005, vol, 5, pp. 1306-1311.

[25] X. Roynard, J.-E. Deschaud, and F. Goulette, "Fast and robust segmentation and classification for change detection in urban point clouds," ISPRS 2016-XXIII ISPRS Congress, 2016.

[26] A. Serna, and B. Marcotegui, "Detection, segmentation and classification of 3D urban objects using mathematical morphology and supervised learning," ISPRS Journal of Photogrammetry and Remote Sensing, vol. 93, pp. 243-255, 2014.

[27] A. Golovinskiy, V.G. Kim, and T. Funkhouser, "Shape-based recognition of 3D point clouds in urban environments," 2009 IEEE 12th International Conference on Computer Vision, IEEE, 2009, pp. 2154-2161.

[28] R. Schnabel, R. Wahl, and R. Klein, "Efficient RANSAC for point-cloud shape detection," Computer graphics forum, Oxford, UK: Blackwell Publishing Ltd, 2007, vol. 26, no. 2, pp. 214-226.

[29] D.H. Ballard, "Generalizing the Hough transform to detect arbitrary shapes," Pattern recognition, vol. 13, no. 2, pp. 111-122, 1981.

[30] V. Sanchez, and A. Zakhor, "Planar 3D modeling of building interiors from point cloud data," 2012 19th IEEE International Conference on Image Processing, IEEE, 2012, pp. 1777-1780.

[31] G.T. Michailidis, R. Pajarola, "Bayesian graph-cut optimization for wall surfaces reconstruction in indoor environments," The Visual Computer, vol. 33 , no. 10, pp. 1347-1355, 2017.

[32] C. Liu, J. Wu, and Y. Furukawa, "FloorNet: A Unified Framework for Floorplan Reconstruction from 3D Scans," In Proceedings of the European Conference on Computer Vision (ECCV), 2018, pp. 201-217.

[33] Y.B. Lin, C. Wang, B.L. Chen, D.W. Zai, and J. Li, "Facet segmentation-based line segment extraction for large-scale point clouds," IEEE Transactions on Geoscience and Remote Sensing, vol. 55, no. 9, pp. 4839-4854, 2017.

[34] A. Boulch, M. de La Gorce, and R. Marlet, "Piecewise - planar 3D reconstruction with edge and corner regularization," Computer Graphics Forum, 2014, vol. 33, no. 5, pp. 55-64.

[35] F. Lafarge, and P. Alliez, "Surface reconstruction through point set structuring," Computer Graphics Forum, Oxford, UK: Blackwell Publishing Ltd, 2013, vol. 32, no. 2pt2, pp. 225-234.

[36] M. L. Li, P. Wonka, and L.L. Nan, "Manhattan-world urban reconstruction from point clouds," European Conference on Computer Vision, Springer, Cham, 2016, pp. 54-69.

[37] S. Oesau, F. Lafarge, and P. Alliez, "Indoor scene reconstruction using feature sensitive primitive extraction and graph-cut," ISPRS Journal of Photogrammetry and Remote Sensing, vol. 90, pp. 68-82, 2014.

[38] L.L. Nan, and P. Wonka, "Polyfit: Polygonal surface reconstruction from point clouds," in Proceedings of the IEEE Conference on Computer Vision and Pattern Recognition, 2017, pp. 2353-2361.

[39] S. Ochmann, R. Vock, R. Wessel, M. Tamke, and R. Klein, "Automatic generation of structural building descriptions from 3d point cloud scans," 2014 International Conference on Computer Graphics Theory and Applications (GRAPP), IEEE, 2014, pp. 1-8.

[40] C. Mura, O. Mattausch, A.J. Villanueva, E. Gobbetti, and R. Pajarola, "Automatic room detection and reconstruction in cluttered indoor environments with complex room layouts," Computers \& Graphics, vol. 44, pp. 20-32, 2014

[41] S. Ikehata, H. Yang, and Y. Furukawa, "Structured indoor modeling," Proceedings of the IEEE International Conference on Computer Vision, 2015, pp. 1323-1331.

[42] J. X. Xiao, and Y. Furukawa, "Reconstructing the world's museums," International journal of computer vision, vol. 110, no. 3, pp. 243-258, 2014.

[43] K. Khoshelham, and L. Díaz-Vilariño, "3D Modelling of Interior Spaces: Learning the Language of Indoor Architecture," The International Archives of
Photogrammetry, Remote Sensing and Spatial Information Sciences, vol. 40, no. 5 , pp. 321, 2014

[44] L. Díaz-Vilariño, L. González-de Santos, E. Verbree, G. Michailidou, and S. Zlatanova, "From point cloud to 3D isovists in indoor environments," International Archives of the Photogrammetry, Remote Sensing \& Spatial Information Sciences, vol. 42, no. 4, 2018.

[45] A. Mizushima, and R. Lu, "An image segmentation method for apple sorting and grading using support vector machine and Otsu's method," Computers and Electronics in Agriculture, vol. 94, pp. 29-37, 2013.

[46] Y. Boykov, and V. Kolmogorov, "An experimental comparison of min-cut/max-flow algorithms for energy minimization in vision," IEEE Transactions on Pattern Analysis and Machine Intelligence, vol. 26, no. 9, pp. 1124-1137, 2004

[47] V. Kolmogorov, and R. Zabin, "What energy functions can be minimized via graph cuts?," IEEE Transactions on Pattern Analysis \& Machine Intelligence, vol. 26, no.2, pp. 147-159, 2002.

[48] Y. Boykov, O. Veksler, and R. Zabih, "Fast Approximate Energy Minimization via Graph Cuts," Pattern Analysis \& Machine Intelligence IEEE Transactions on, vol. 23, no. 11, pp. 1222-1239, 2002.

[49] K. Khoshelham, L. Díaz Vilariño, M. Peter, Z. Kang, and D. Acharya, "The Isprs Benchmark on Indoor Modelling," ISPRS - International Archives of the Photogrammetry, Remote Sensing and Spatial Information Sciences, vol. 42, pp. 367-372, 2017.

[50] B. Wu, B.L. Yu, Q.S. Wu, S.J. Yao, F. Zhao, W.Q. Mao, and J.P. Wu, "A Graph-Based Approach for 3D Building Model Reconstruction from Airborne LiDAR Point Clouds," Remote Sensing, vol. 9, no.1, pp. 92, 2017.

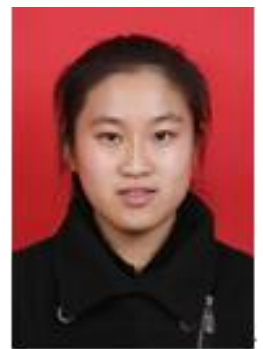

Yang Cui received the B.S. and M.S. degrees in school of surveying and geographical sciences from Liaoning Engineering Technology University, China, in 2013 and 2016. She is currently reading doctorate of the college of information engineering, Shenzhen University. Her research interests include laser scanning and computer graphics, with a focus on 3-D indoor modeling.

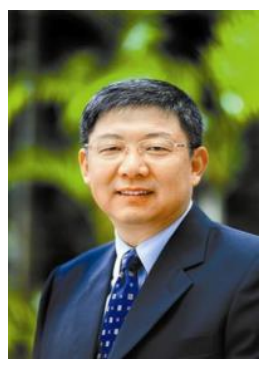

Qing quan Li received the B.S. degree in engineering survey, the M.S. degree, and the Ph.D. degree in photogrammetry and remote sensing from Wuhan University, China, in 1984, 1988, and 1998, respectively. Since 1998, he has been a Professor with the State Key Laboratory of Information Engineering in Surveying, Mapping and Remote Sensing, Wuhan University. From 2000 to 2012, he was the Vice President of Wuhan University. He is currently the President of Shenzhen University. He is the author of five books and over 400 articles, and holding 13 software copyrights and nine invention patents. His research interests include intelligent traffic systems, urban informatics, and smart city. He is a Chief Editor of the Journal of Geomatics, and an Associate Editor of the Journal Acta Geodaetica et Cartographica Sinica and Geomatics and Information Science of Wuhan University.

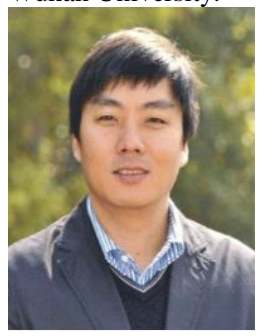

Bi sheng Yang received the B.S. degree in engineering survey, the M.S. degree, and the Ph.D. degree in photogrammetry and remote sensing from Wuhan University, China, in 1996, 1999, and 2002, respectively. From 2002 to 2006, he held a post-doctoral position at the University of Zurich, Switzerland. Since 2007, he has been a Professor with the State Key Laboratory of Information Engineering in Surveying, Mapping and Remote Sensing, Wuhan University, where he is currently the Director of the $3 \mathrm{~S}$ and Network Communication Laboratory. He has hosted a project of the National High Technology Research and Development Program, a key project of the Ministry of Education, and four National Scientific Research Foundation Project of China. His main research interests comprise 3-D geographic information systems, urban modeling, and digital city. He was a Guest Editor of the ISPRS Journal of Photogrammetry and Remote Sensing, and Computers \& Geosciences. 


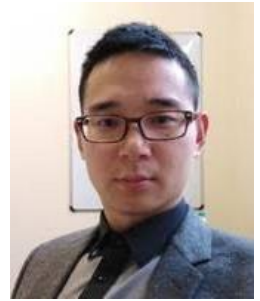

Wen Xiao received the B.S. degree in geodesy and geomatics from the Wuhan University, Wuhan, China, in 2010, the M.S. (cum laude) degree in geoinformatics from ITC, University of Twente, Enschede, The Netherlands, in 2012, and the Ph.D. degree in geoinformation science and technology from IGN, Université Paris-EST, Paris, France, in 2015. Since 2016, he has been a Lecturer and University Research Fellow at Newcastle University, Newcastle upon Tyne, U.K. He is currently a Council Member of RSPSoc. His research interests include 3D mapping, laser scanning, photogrammetric computer vision, and their applications in urban and natural environments.

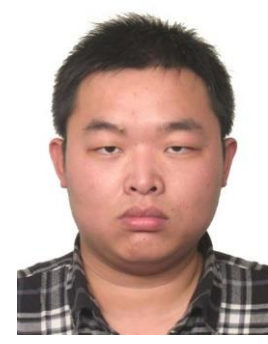

Chi Chen received his B.E. and Ph.D. degrees in Remote Sensing and Photogrammetry from the Wuhan University in 2010 and 2016. He is currently an associate professor of the State Key Laboratory of Information Engineering in Surveying, Mapping and Remote Sensing (LIESMARS), Wuhan University. His research interests include $3 \mathrm{D}$ image/LiDAR point clouds processing, mixed reality, computational photography and remote sensing methods for power industry.

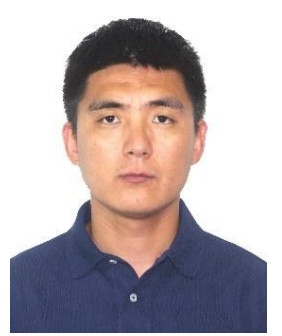

Zhen Dong received his B.E. and Ph.D. degrees in Remote Sensing and Photogrammetry from the Wuhan University in 2011 and 2018. He is currently a postdoctoral of the State Key Laboratory of Information Engineering in Surveying, Mapping and Remote Sensing (LIESMARS), Wuhan University. His research interests include $3 \mathrm{D}$ vision, point clouds processing and deep learning. 